\title{
Integral Inequalities of Hermite-Hadamard Type for $\lambda$-MT-Convex Function
}

\author{
Opeyemi Omotoyinbo, Adesanmi Mogbademu* and Peter Olanipekun
}

(Communicated by Nihal YILMAZ ÖZGÜR)

\begin{abstract}
In this paper, we establish some Hermite-Hadamard type Integral inequalities for a new class of convex function called $\lambda$-MT-convex function. Our results generalize and extend some existing results in literature.
\end{abstract}

Keywords: Hermite-Hadamard integral Inequalities; MT-convex function; $\lambda$-MT-convex function.

AMS Subject Classification (2010): Primary: 26D15; Secondary: $26 A 51$.

${ }^{*}$ Corresponding author

\section{Introduction}

In this section, some definitions and results used in this paper are presented.

Let $f: I \subseteq R \rightarrow R$ be a convex function defined on the interval $I=[a, b]$ of the real numbers and let $a, b \in[c, d]$ where $a<b$. Then, the following double inequality

$$
f\left(\frac{a+b}{2}\right) \leq \frac{1}{b-a} \int_{a}^{b} f(x) d x \leq \frac{f(a)+f(b)}{2}
$$

is known in the literature as the Hermite-Hadamard Integral Inequality [7]. The inequality, often referred to as the first fundamental result for convex functions with a natural geometrical interpretation and many applications, has attracted and continues to attract much interest in the field of Optimization, Mathematics and Engineering since its establishment in 1881 (see [2]). A good number of research papers and texts have been written on (1.1), providing new proofs, expositions, noteworthy extensions, generalizations and numerous applications (see [5]).

We give some definitions relating to convex functions below:

Definition 1.1. [2] A function $f: I \rightarrow R$ is said to be convex, if for every $x, y \in I$ and $t \in[0,1]$, we have

$$
f(t x+(1-t) y) \leq t f(x)+(1-t) f(y) .
$$

Definition 1.2. [4] A function $f:[0, b] \rightarrow R$ is said to be $m$-convex, where $m \in[0,1]$, if for every $x, y \in[0, b]$, and $t \in[0,1]$, we have

$$
f(t x+m(1-t) y) \leq t f(x)+m(1-t) f(y) .
$$

In [11], Tunc and Yildirim defined the class of MT-convex functions as follows.

Definition 1.3. [11] A function $f: I \subseteq R \rightarrow R$ is said to belong to the class $M T(I)$ if $f$ is nonnegative and $\forall x, y \in I$ and $t \in(0,1)$ satisfies the inequality

$$
f(t x+(1-t) y) \leq \frac{\sqrt{t}}{2 \sqrt{1-t}} f(x)+\frac{\sqrt{1-t}}{2 \sqrt{t}} f(y) .
$$

Received : 11-September-2014, Accepted : 12-January-2016 
Recently, Omotoyinbo and Mogbademu [9] introduced a new class of convex function as follows.

Definition 1.4. [9] A function $f: I \subseteq R \rightarrow R$ is said to belong to the class $m-M T(I)$ if $f$ is nonnegative and $\forall x, y \in I$ and $t \in(0,1)$, with $m \in[0,1]$ satisfies the inequality

$$
f(t x+m(1-t) y) \leq \frac{\sqrt{t}}{2 \sqrt{1-t}} f(x)+\frac{m \sqrt{1-t}}{2 \sqrt{t}} f(y) .
$$

Recently, Tunc and Yildirim [11] obtained the following two new inequalities of Hermite-Hadamard type for the class of MT-convex functions.

Theorem 1.1. Let $f \in M T(I), a, b \in I$ with $a<b$ and $f \in L_{1}[a, b]$. Then, one has the inequality

$$
f\left(\frac{a+b}{2}\right) \leq \frac{1}{b-a} \int_{a}^{b} f(x) d x
$$

and

$$
\frac{2}{b-a} \int_{a}^{b} \tau(x) f(x) d x \leq \frac{f(a)+f(b)}{2}
$$

where $\tau(x)=\frac{\sqrt{(b-a)(x-a)}}{b-a}, x \in[a, b]$.

In [12], Tunc et al. established some Hermite-Hadamard inequalities for MT-convex functions. Indeed, they proved the following:

Theorem 1.2. Let $f:[a, b] \subseteq R \rightarrow R$ be nonnegative MT-convex function and $f \in L_{1}[a, b]$. Then,

$$
\frac{1}{b-a} \int_{a}^{b} f(x) d x \leq \frac{\pi}{4}(f(a)+f(b)) .
$$

Theorem 1.3. Let $f, g \in[a, b] \rightarrow R$ be two nonnegative MT-convex functions and $f, g \in L_{1}[a, b]$. Then,

$$
\frac{8}{3} f\left(\frac{a+b}{2}\right) g\left(\frac{a+b}{2}\right) \leq M(a, b)+N(a, b),
$$

where $M(a, b)=f(a) g(a)+f(b) g(b), N(a, b)=f(a) g(b)+f(b) g(a)$.

Theorem 1.4. Let $f, g:[a, b] \subseteq R \rightarrow R$ two nonnegative MT-convex functions and $f, g \in L_{1}([a, b])$. Then, $f\left(\frac{a+b}{2}\right)(g(a)+g(b))+g\left(\frac{a+\bar{b}}{2}\right)(f(a)+f(b))$

$$
\leq \frac{16}{3 \pi} f\left(\frac{a+b}{2}\right) g\left(\frac{a+b}{2}\right)+2(f(a)+f(b))(g(a)+g(b)) .
$$

Motivated by the results of Dragomir et al. [3], Tunc and Yildirim [11], Omotoyinbo and Mogbademu [8], [9] and Tunc et al. [12], we introduce and define the following new class of convex functions.

Definition 1.5. A nonnegative function $f: I \subseteq R \rightarrow R$ is said to be a $\lambda$-MT-convex function or said to belong to the class $\lambda-M T(I)$ if $\forall x, y \in I, \lambda \in\left(0, \frac{1}{2}\right]$ and $t \in(0,1)$ the following inequality is satisfied

$$
f(t x+(1-t) y) \leq \frac{\sqrt{t}}{2 \sqrt{1-t}} f(x)+\frac{(1-\lambda) \sqrt{1-t}}{2 \lambda \sqrt{t}} f(y) .
$$

Remark 1.1. An example to illustrate this type of function is:

$f:[1,2] \rightarrow R, f(x)=x^{k}, k \in\left(0, \frac{1}{1000}\right)$.

By choosing $I=[1,2], \lambda \in\left[\frac{1}{3}, \frac{2}{5}\right], t=0.5, x=1, y=2$, inequality (1.10) is satisfied. Thus, $f$ is $\lambda$-MT-convex.

The purpose of this paper is to establish some new Hermite-Hadamard type integral inequalities for $\lambda$-MTconvex function, thereby extending known results in literature, using simple analytical techniques. 


\section{Main Results}

Theorem 2.1. Let $f, g \in[a, b] \rightarrow R$ be two nonnegative $\lambda-M T$-convex functions and $f, g \in L_{1}([a, b])$ with $a, b \in I$ and $a<b$. Then

$$
8 \lambda^{2} f\left(\frac{a+b}{2}\right) g\left(\frac{a+b}{2}\right) \leq\left(\lambda^{2}-\lambda+1\right)(M(a, b)+N(a, b)),
$$

where $M(a, b)=f(a) g(a)+f(b) g(b), N(a, b)=f(a) g(b)+f(b) g(a)$.

Proof. Since $f$ and $g$ are $\lambda-M T$-convex, then from Definition 1.5,

$$
\begin{aligned}
f\left(\frac{a+b}{2}\right) \leq & \frac{\sqrt{t}}{2 \sqrt{1-t}} f(a)+\frac{(1-\lambda) \sqrt{1-t}}{2 \lambda \sqrt{t}} f(b) \\
& \leq \frac{1}{2}\left(\frac{\sqrt{t}}{2 \sqrt{1-t}}+\frac{(1-\lambda) \sqrt{1-t}}{2 \lambda \sqrt{t}}\right)(f(a)+f(b))
\end{aligned}
$$

and

$$
\begin{aligned}
g\left(\frac{a+b}{2}\right) \leq & \frac{\sqrt{t}}{2 \sqrt{1-t}} g(a)+\frac{(1-\lambda) \sqrt{1-t}}{2 \lambda \sqrt{t}} g(b) \\
& \leq \frac{1}{2}\left(\frac{\sqrt{t}}{2 \sqrt{1-t}}+\frac{(1-\lambda) \sqrt{1-t}}{2 \lambda \sqrt{t}}\right)(g(a)+g(b)) .
\end{aligned}
$$

Multiplying (2.2) and (2.3), we obtain

$$
\begin{aligned}
f\left(\frac{a+b}{2}\right) g\left(\frac{a+b}{2}\right) & \leq\left[\frac{1}{4}\left(\frac{\sqrt{t}}{\sqrt{1-t}}+\frac{(1-\lambda) \sqrt{1-t}}{\lambda \sqrt{t}}\right)\right]^{2}(f(a)+f(b))(g(a)+g(b)) \\
& =\frac{1}{16}\left(\frac{\lambda^{2} t^{2}+2 \lambda(1-\lambda) t(1-t)+(1-\lambda)^{2}(1-t)^{2}}{\lambda^{2} t(1-t)}\right)(f(a)+f(b))(g(a)+g(b)) .
\end{aligned}
$$

It is easy to see that (2.4) gives

$$
\begin{aligned}
16 \lambda^{2} t(1-t) f\left(\frac{a+b}{2}\right) g\left(\frac{a+b}{2}\right) \leq & \left(\lambda^{2} t^{2}+2 \lambda(1-\lambda) t(1-t)\right. \\
& \left.+(1-\lambda)^{2}(1-t)^{2}\right)(f(a)+f(b))(g(a)+g(b)) .
\end{aligned}
$$

Integrating both sides of $(2.5)$ wrt $t$ over $[0,1]$ to get

$$
\begin{aligned}
16 \lambda^{2} f\left(\frac{a+b}{2}\right) g\left(\frac{a+b}{2}\right) \int_{0}^{1} t(1-t) d t \leq & (f(a)+f(b))(g(a)+g(b))\left(\lambda^{2} \int_{0}^{1} t^{2} d t\right. \\
& +2 \lambda(1-\lambda) \int_{0}^{1} t(1-t) d t \\
& \left.+(1-\lambda)^{2} \int_{0}^{1}(1-t)^{2} d t\right) .
\end{aligned}
$$

Substituting $\int_{0}^{1} t(1-t) d t=\frac{1}{6}, \int_{0}^{1} t^{2} d t=\int_{0}^{1}(1-t)^{2} d t=\frac{1}{3}$ in (2.5) and simplifying further gives the inequality (2.1).

Remark 2.1. Setting $\lambda=\frac{\sqrt{3}}{2}$ in Theorem 2.1 gives

$$
\begin{aligned}
\frac{8}{3} f\left(\frac{a+b}{2}\right) g\left(\frac{a+b}{2}\right) & \leq\left(\frac{4-\sqrt{3}}{3}\right)(M(a, b)+N(a, b)) \\
& \leq M(a, b)+N(a, b)
\end{aligned}
$$

which is Theorem 2.5 of Tunc et al. [12].

Theorem 2.2. Let $f, g:[a, b] \subseteq R \rightarrow R$ be two nonnegative $\lambda$-MT-convex functions and $f, g \in L_{1}([a, b])$ with $a, b \in I$ where $a<b$. Then

$$
\begin{aligned}
f\left(\frac{a+b}{2}\right)(g(a)+g(b))+g\left(\frac{a+b}{2}\right)(f(a)+f(b)) & \leq \frac{16 \lambda}{3 \pi} f\left(\frac{a+b}{2}\right) g\left(\frac{a+b}{2}\right) \\
& +\frac{4}{3 \lambda \pi}\left(\lambda^{4}-2 \lambda^{3}+2 \lambda^{2}+\lambda+1\right) \\
& \times(M(a, b)+N(a, b))
\end{aligned}
$$

where $M(a, b)=f(a) g(a)+f(b) g(b), N(a, b)=f(a) g(b)+f(b) g(a)$. 
Proof. Since $f, g \in \lambda-M T(I)$, then

$$
\begin{aligned}
& f\left(\frac{a+b}{2}\right) \leq \frac{\sqrt{t}}{2 \sqrt{1-t}} f(a)+\frac{(1-\lambda) \sqrt{1-t}}{2 \lambda \sqrt{t}} f(b) \\
& \leq \frac{1}{2}\left(\frac{\sqrt{t}}{2 \sqrt{1-t}}+\frac{(1-\lambda) \sqrt{1-t}}{2 \lambda \sqrt{t}}\right)(f(a)+f(b)), \\
& g\left(\frac{a+b}{2}\right) \leq \frac{\sqrt{t}}{2 \sqrt{1-t}} g(a)+\frac{(1-\lambda) \sqrt{1-t}}{2 \lambda \sqrt{t}} g(b) \\
& \quad \leq \frac{1}{2}\left(\frac{\sqrt{t}}{2 \sqrt{1-t}}+\frac{(1-\lambda) \sqrt{1-t}}{2 \lambda \sqrt{t}}\right)(g(a)+g(b)) .
\end{aligned}
$$

Recall: For $p, q, r, s, t \in R^{+}$, if $p \leq s$, and $r \leq q$ then $p q+r s \leq p s+q r$.

$$
\begin{aligned}
& \frac{1}{4} f\left(\frac{a+b}{2}\right)\left(\frac{\sqrt{t}}{\sqrt{1-t}}+\frac{(1-\lambda) \sqrt{1-t}}{\lambda \sqrt{t}}\right)(g(a)+g(b)) \\
&+ \frac{1}{4} g\left(\frac{a+b}{2}\right)\left(\frac{\sqrt{t}}{\sqrt{1-t}}+\frac{(1-\lambda) \sqrt{1-t}}{\lambda \sqrt{t}}\right)(f(a)+f(b)) \\
& \leq f\left(\frac{a+b}{2}\right) g\left(\frac{a+b}{2}\right)+\left[\frac{1}{4}\left(\frac{\sqrt{t}}{\sqrt{1-t}}+\frac{(1-\lambda) \sqrt{1-t}}{\lambda \sqrt{t}}\right)\right]^{2}(f(a)+f(b))(g(a)+g(b)) .
\end{aligned}
$$

Simplifying (2.9), we get

$$
\begin{aligned}
& \frac{1}{4} f\left(\frac{a+b}{2}\right)\left(\frac{\sqrt{t}}{\sqrt{1-t}}+\frac{(1-\lambda) \sqrt{1-t}}{\lambda \sqrt{t}}\right)(g(a)+g(b))+\frac{1}{4} g\left(\frac{a+b}{2}\right)\left(\frac{\sqrt{t}}{\sqrt{1-t}}+\frac{(1-\lambda) \sqrt{1-t}}{\lambda \sqrt{t}}\right)(f(a)+f(b)) \\
& \leq f\left(\frac{a+b}{2}\right) g\left(\frac{a+b}{2}\right)+\left(\frac{\lambda^{2} t^{2}+2 \lambda(1-\lambda) t(1-t)+\lambda^{2}(1-\lambda)^{2}(1-t)^{2}}{16 \lambda^{2} t(1-t)}\right)(f(a)+f(b))(g(a)+g(b)) .
\end{aligned}
$$

Multiplying both sides of the inequality (2.10) by $16 \lambda^{2} t(1-t)$, gives

$$
\begin{aligned}
& 4 f\left(\frac{a+b}{2}\right)\left(\lambda^{2} t^{\frac{3}{2}}(1-t)^{\frac{1}{2}}+\lambda(1-\lambda) t^{\frac{1}{2}}(1-t)^{\frac{3}{2}}\right)(g(a)+g(b)) \\
& +4 g\left(\frac{a+b}{2}\right)\left(\lambda^{2} t^{\frac{3}{2}}(1-t)^{\frac{1}{2}}+\lambda(1-\lambda) t^{\frac{1}{2}}(1-t)^{\frac{3}{2}}\right)(f(a)+f(b)) \\
& \leq 16 \lambda^{2} t(1-t) f\left(\frac{a+b}{2}\right) g\left(\frac{a+b}{2}\right)+\left(\lambda^{2} t^{2}+2(1-\lambda) t(1-t)\right. \\
& \left.+\lambda^{2}(1-\lambda)^{2}(1-t)^{2}\right)(f(a)+f(b))(g(a)+g(b)) .
\end{aligned}
$$

Integrating both sides of (2.11) wrt $t$ over $[0,1]$,

$$
\begin{gathered}
4 f\left(\frac{a+b}{2}\right)\left(\lambda^{2} \int_{0}^{1} t^{\frac{3}{2}}(1-t)^{\frac{1}{2}} d t+\lambda(1-\lambda) \int_{0}^{1} t^{\frac{1}{2}}(1-t)^{\frac{3}{2}} d t\right)(g(a)+g(b) \\
+4 g\left(\frac{a+b}{2}\right)\left(\lambda^{2} \int_{0}^{1} t^{\frac{3}{2}}(1-t)^{\frac{1}{2}} d t+\lambda(1-\lambda) \int_{0}^{1} t^{\frac{1}{2}}(1-t)^{\frac{3}{2}}\right)(f(a)+f(b)) \\
\leq 16 \lambda^{2} f\left(\frac{a+b}{2}\right) g\left(\frac{a+b}{2}\right) \int_{0}^{1} t(1-t) d t \\
+\left(\lambda^{2} \int_{0}^{1} t^{2} d t+2(1-\lambda) \int_{0}^{1} t(1-t) d t+\lambda^{2}(1-\lambda)^{2} \int_{0}^{1}(1-t)^{2} d t\right)(f(a)+f(b))(g(a)+g(b)) .
\end{gathered}
$$

Substitute the following equalities in (2.12),

$$
\begin{gathered}
\int_{0}^{1} t^{\frac{3}{2}}(1-t)^{\frac{1}{2}} d t=\int_{0}^{1} t^{\frac{1}{2}}(1-t)^{\frac{3}{2}} d t=\frac{\pi}{16} \\
\int_{0}^{1} t(1-t) d t=\frac{1}{6}, \int_{0}^{1} t^{2} d t=\int_{0}^{1}(1-t)^{2} d t=\frac{1}{3} .
\end{gathered}
$$

This completes the proof. 
Theorem 2.3. Let $f:[a, b] \subseteq R \rightarrow R$ be a nonnegative $\lambda-M T$-convex functions and $f \in L_{1}([a, b])$ with $a, b \in I$ where $a<b$. Then

$$
\frac{1}{\left(\left(\frac{\lambda}{1-\lambda}\right) b-a\right)} \int_{a}^{\left(\frac{\lambda}{1-\lambda}\right) b} f(x) d x+\frac{1}{\left(b-\left(\frac{\lambda}{1-\lambda}\right) a\right)} \int_{\left(\frac{\lambda}{1-\lambda}\right) a}^{b} f(y) d y \leq \frac{\pi}{4}\left(\frac{1}{\lambda}\right)(f(a)+f(b))
$$

Proof. Since $f \in \lambda-M T(I)$. Then, we can write

$$
\begin{aligned}
& f\left(t a+\frac{\lambda}{1-\lambda}(1-t) b\right) \leq \frac{\sqrt{t}}{2 \sqrt{1-t}} f(a)+\frac{(1-\lambda) \sqrt{1-t}}{2 \lambda \sqrt{t}} f(b), \\
& f\left(t b+\frac{\lambda}{1-\lambda}(1-t) a\right) \leq \frac{\sqrt{t}}{2 \sqrt{1-t}} f(b)+\frac{(1-\lambda) \sqrt{1-t}}{2 \lambda \sqrt{t}} f(a) .
\end{aligned}
$$

Adding (2.13) and (2.14) gives

$$
f\left(t a+\frac{\lambda}{1-\lambda}(1-t) b\right)+f\left(t b+\frac{\lambda}{1-\lambda}(1-t) a\right) \leq \frac{1}{2}\left(\frac{\sqrt{t}}{\sqrt{1-t}}+\frac{(1-\lambda) \sqrt{1-t}}{\lambda \sqrt{t}}\right)(f(a)+f(b)) .
$$

Integrating both sides of (2.15) wrt to $t$,

$$
\begin{aligned}
& \int_{0}^{1} f\left(t a+\frac{\lambda}{1-\lambda}(1-t) b\right) d t+\int_{0}^{1} f\left(t b+\frac{\lambda}{1-\lambda}(1-t) a\right) d t \\
& \leq \frac{1}{2}\left(\int_{0}^{1} t^{\frac{1}{2}}(1-t)^{\frac{1}{2}} d t+\frac{1-\lambda}{\lambda} \int_{0}^{1} t^{\frac{1}{2}}(1-t)^{\frac{1}{2}} d t\right)(f(a)+f(b)) .
\end{aligned}
$$

Substituting $x=t a+\frac{\lambda}{1-\lambda}(1-t) b, d x=\left(a-\left(\frac{\lambda}{1-\lambda}\right) b\right) d t$,

$$
y=t b+\frac{\lambda}{1-\lambda}(1-t) a, d y=\left(b-\left(\frac{\lambda}{1-\lambda}\right) a\right) d t
$$

where $\int_{0}^{1} t^{-\frac{1}{2}}(1-t)^{\frac{1}{2}} d t=\int_{0}^{1} t^{\frac{1}{2}}(1-t)^{-\frac{1}{2}} d t=\frac{\pi}{2}$ into (2.16) and simplifying to get:

$$
\frac{1}{\left(\left(\frac{\lambda}{1-\lambda}\right) b-a\right)} \int_{a}^{\left(\frac{\lambda}{1-\lambda}\right) b} f(x) d x+\frac{1}{\left(b-\left(\frac{\lambda}{1-\lambda}\right) a\right)} \int_{\left(\frac{\lambda}{1-\lambda}\right) a}^{b} f(y) d y \leq \frac{\pi}{4}\left(\frac{1}{\lambda}\right)(f(a)+f(b)) .
$$

Hence, the proof is completed.

Remark 2.2. Let $f:[a, b] \subseteq R \rightarrow R$ be a nonnegative MT-convex function and $f \in L_{1}[a, b]$. By choosing $\lambda=\frac{1}{2}$ and $x=y$ we obtain

$$
\frac{1}{b-a} \int_{a}^{b} f(x) d x \leq \frac{\pi}{4}(f(a)+f(b)),
$$

which is Theorem 2.3 of Tunc et al. [12].

Remark 2.3. Alternatively, we can integrate directly either of the $\lambda-M T$-convex functions

$$
f\left(t a+\frac{\lambda}{1-\lambda}(1-t) b\right) \leq \frac{\sqrt{t}}{2 \sqrt{1-t}} f(a)+\frac{(1-\lambda) \sqrt{1-t}}{2 \lambda \sqrt{t}} f(b)
$$

or

$$
f\left(t b+\frac{\lambda}{1-\lambda}(1-t) a\right) \leq \frac{\sqrt{t}}{2 \sqrt{1-t}} f(b)+\frac{(1-\lambda) \sqrt{1-t}}{2 \lambda \sqrt{t}} f(a)
$$

to obtain

$$
\frac{1}{\left(\left(\frac{\lambda}{1-\lambda}\right) b-a\right)} \int_{a}^{\left(\frac{\lambda}{1-\lambda}\right) b} f(x) d x \leq \frac{\pi}{4}\left(f(a)+\left(\frac{1-\lambda}{\lambda}\right) f(b)\right)
$$

or

$$
\frac{1}{\left(\left(\frac{\lambda}{1-\lambda}\right) a-b\right)} \int_{b}^{\left(\frac{\lambda}{1-\lambda}\right) a} f(x) d x \leq \frac{\pi}{4}\left(f(b)+\left(\frac{1-\lambda}{\lambda}\right) f(a)\right) .
$$


Theorem 2.4. Let $f:[a, b] \subseteq R \rightarrow R$ be a nonnegative $\lambda-M T$-convex functions and $f \in L_{1}([a, b])$ with $a, b \in I$ where $a<b$. Then

$$
f\left(\frac{a+\left(\frac{\lambda}{1-\lambda}\right) b}{2}\right) \leq \frac{1}{2}\left(\frac{1}{\left(\left(\frac{\lambda}{1-\lambda}\right) b-a\right)} \int_{a}^{\left(\frac{\lambda}{1-\lambda}\right) b} f(x) d x+\frac{1}{\left(b-\left(\frac{\lambda}{1-\lambda}\right) a\right)} \int_{\left(\frac{\lambda}{1-\lambda}\right) a}^{b} f(y) d y\right) .
$$

Proof. Since $f \in \lambda-M T(I)$. Then, for all $x, y \in I$,

$$
f\left(t a+\frac{\lambda}{1-\lambda}(1-t) b\right) \leq \frac{\sqrt{t}}{2 \sqrt{1-t}} f(a)+\frac{(1-\lambda) \sqrt{1-t}}{2 \lambda \sqrt{t}} f(b) .
$$

Substituting $t=\frac{1}{2}$ in inequality (2.18), we have,

$$
f\left(\frac{a+\left(\frac{\lambda}{1-\lambda}\right) b}{2}\right) \leq \frac{f(a)+\left(\frac{\lambda}{1-\lambda}\right) f(b)}{2}
$$

That is, with $x=t a+\frac{\lambda}{1-\lambda}(1-t) b, d x=\left(a-\left(\frac{\lambda}{1-\lambda}\right) b\right) d t$,

$$
y=\frac{\lambda}{1-\lambda}(1-t) a+t b, d y=\left(b-\left(\frac{\lambda}{1-\lambda}\right) a\right) d t,
$$

where $\int_{0}^{1} t^{-\frac{1}{2}}(1-t)^{\frac{1}{2}} d t=\int_{0}^{1} t^{\frac{1}{2}}(1-t)^{-\frac{1}{2}} d t=\frac{\pi}{2}$.

$$
f\left(\frac{a+\left(\frac{\lambda}{1-\lambda}\right) b}{2}\right) \leq \frac{1}{2}\left(\int_{0}^{1} f\left(t a+\frac{\lambda}{1-\lambda}(1-t) b\right) d t+\int_{0}^{1} f\left(\frac{\lambda}{1-\lambda}(1-t) a+t b\right) d t\right) .
$$

Further simlification of inequality (2.19) completely gives (2.17). Hence, the proof is completed.

Remark 2.4. Setting $\lambda=\frac{1}{2}$, in (2.17) we obtain

$$
f\left(\frac{a+b}{2}\right) \leq \frac{1}{b-a} \int_{a}^{b} f(x) d x
$$

which is the first part of Hadamard's inequality and Theorem 2a of Tunc and Yildirim[11].

Theorem 2.5. Let $f, g:[a, b] \subseteq R \rightarrow R$ be two nonnegative $\lambda-M T$-convex functions and $f \in L_{1}([a, b])$ with $a, b \in I$ where $a<b$. Then

$$
\begin{gathered}
g(a) \frac{\lambda^{2}}{\left(\left(\frac{\lambda}{1-\lambda}\right) b-a\right)^{3}} \int_{a}^{\left(\frac{\lambda}{1-\lambda}\right) b}\left(\left(\frac{\lambda}{1-\lambda}\right) b-x\right)^{\frac{3}{2}}(x-a)^{\frac{1}{2}} f(x) d x \\
+g(b) \frac{\lambda(1-\lambda)}{\left(\left(\frac{\lambda}{1-\lambda}\right) b-a\right)^{3}} \int_{a}^{\left(\frac{\lambda}{1-\lambda}\right) b}\left(\left(\frac{\lambda}{1-\lambda}\right) b-x\right)^{\frac{1}{2}}(x-a)^{\frac{3}{2}} f(x) d x \\
f(a) \frac{\lambda^{2}}{\left(\left(\frac{\lambda}{1-\lambda}\right) b-a\right)^{3}} \int_{a}^{\left(\frac{\lambda}{1-\lambda}\right) b}\left(\left(\frac{\lambda}{1-\lambda}\right) b-x\right)^{\frac{3}{2}}(x-a)^{\frac{1}{2}} g(x) d x \\
+f(b) \frac{\lambda(1-\lambda)}{\left(\left(\frac{\lambda}{1-\lambda}\right) b-a\right)^{3}} \int_{a}^{\left(\frac{\lambda}{1-\lambda}\right) b}\left(\left(\frac{\lambda}{1-\lambda}\right) b-x\right)^{\frac{1}{2}}(x-a)^{\frac{3}{2}} g(x) d x \\
\leq \frac{1}{2}\left(\frac{\lambda^{3}}{3} f(a) g(a)+\frac{(1-\lambda)^{2}}{3} f(b) g(b)+\frac{\lambda(1-\lambda)}{6}(f(a) g(b)+f(b) g(a))\right) \\
+\frac{2 \lambda^{2}}{\left(\left(\frac{\lambda}{1-\lambda}\right) b-a\right)^{3}} \int_{a}^{\left(\frac{\lambda}{1-\lambda}\right) b}\left(\left(\frac{\lambda}{1-\lambda}\right) b-x\right)(x-a) f(x) g(x) d x
\end{gathered}
$$


Proof. Since $f$ and $g$ are $\lambda-M T$-convex, we can write

$$
\begin{aligned}
& f\left(t a+\frac{\lambda}{1-\lambda}(1-t) b\right) \leq \frac{\sqrt{t}}{2 \sqrt{1-t}} f(a)+\frac{(1-\lambda) \sqrt{1-t}}{2 \lambda \sqrt{t}} f(b), \\
& g\left(t a+\frac{\lambda}{1-\lambda}(1-t) b\right) \leq \frac{\sqrt{t}}{2 \sqrt{1-t}} g(a)+\frac{(1-\lambda) \sqrt{1-t}}{2 \lambda \sqrt{t}} g(b) .
\end{aligned}
$$

Using the basic inequality, $p q+r s \leq p s+q r$, whenever $p \leq s$ and $r \leq q$ for any $p, q, r, s \in R^{+}$we have

$$
\begin{gathered}
f\left(t a+\frac{\lambda}{1-\lambda}(1-t) b\right)\left(\frac{\sqrt{t}}{2 \sqrt{1-t}} g(a)+\frac{(1-\lambda) \sqrt{1-t}}{2 \lambda \sqrt{t}} g(b)\right) \\
+g\left(t a+\frac{\lambda}{1-\lambda}(1-t) b\right)\left(\frac{\sqrt{t}}{2 \sqrt{1-t}} f(a)+\frac{(1-\lambda) \sqrt{1-t}}{2 \lambda \sqrt{t}} f(b)\right) \\
\leq\left(\frac{\sqrt{t}}{2 \sqrt{1-t}} f(a)+\frac{(1-\lambda) \sqrt{1-t}}{2 \lambda \sqrt{t}} f(b)\right)\left(\frac{\sqrt{t}}{2 \sqrt{1-t}} g(a)+\frac{(1-\lambda) \sqrt{1-t}}{2 \lambda \sqrt{t}} g(b)\right) \\
+f\left(t a+\frac{\lambda}{1-\lambda}(1-t) b\right) g\left(t a+\frac{\lambda}{1-\lambda}(1-t) b\right) .
\end{gathered}
$$

This gives

$$
\begin{gathered}
g(a) \frac{\sqrt{t} \sqrt{1-t}}{1-t} f\left(t a+\frac{\lambda}{1-\lambda}(1-t) b\right)+g(b) \frac{(1-\lambda) \sqrt{t} \sqrt{1-t}}{\lambda t} f\left(t a+\frac{\lambda}{1-\lambda}(1-t) b\right) \\
+f(a) \frac{\sqrt{t} \sqrt{1-t}}{1-t} g\left(t a+\frac{\lambda}{1-\lambda}(1-t) b\right)+f(b) \frac{(1-\lambda) \sqrt{t} \sqrt{1-t}}{\lambda t} g\left(t a+\frac{\lambda}{1-\lambda}(1-t) b\right) \\
\leq \frac{1}{2}\left(\frac{t}{1-t} f(a) g(a)+\frac{(1-\lambda)}{\lambda} f(a) g(b)+\frac{(1-\lambda)}{\lambda} f(b) g(a)+\frac{(1-\lambda)^{2}(1-t)}{\lambda^{2} t} f(b) g(b)\right) \\
+2 f\left(t a+\frac{\lambda}{1-\lambda}(1-t) b\right) g\left(t a+\frac{\lambda}{1-\lambda}(1-t) b\right) .
\end{gathered}
$$

It can be eaily seen that (2.21) gives

$$
\begin{gathered}
g(a) \lambda^{2} t \sqrt{t} \sqrt{1-t} f\left(t a+\frac{\lambda}{1-\lambda}(1-t) b\right)+g(b) \lambda(1-\lambda)(1-t) \sqrt{t} \sqrt{1-t} f\left(t a+\frac{\lambda}{1-\lambda}(1-t) b\right) \\
+f(a) \lambda^{2} t \sqrt{t} \sqrt{1-t} g\left(t a+\frac{\lambda}{1-\lambda}(1-t) b\right)+f(b) \lambda(1-\lambda)(1-t) \sqrt{t} \sqrt{1-t} g\left(t a+\frac{\lambda}{1-\lambda}(1-t) b\right) \\
\leq \frac{1}{2}\left(\lambda^{2} t^{2} f(a) g(a)+\lambda(1-\lambda) t(1-t)(f(a) g(b)+f(b) g(a))+(1-\lambda)^{2}(1-t)^{2} f(b) g(b)\right) \\
+2 \lambda^{2} t(1-t)\left(f\left(t a+\frac{\lambda}{1-\lambda}(1-t) b\right) g\left(t a+\frac{\lambda}{1-\lambda}(1-t) b\right)\right)
\end{gathered}
$$

Integrating both sides of (2.22) over $[0,1]$ wrt to $t$,

$$
\begin{gathered}
g(a) \lambda^{2} \int_{0}^{1} t \sqrt{t} \sqrt{1-t} f\left(t a+\frac{\lambda}{1-\lambda}(1-t) b\right) d t \\
+g(b) \lambda(1-\lambda) \int_{0}^{1}(1-t) \sqrt{t} \sqrt{1-t} f\left(t a+\frac{\lambda}{1-\lambda}(1-t) b\right) d t \\
+f(a) \lambda^{2} \int_{0}^{1} t \sqrt{t} \sqrt{1-t} g\left(t a+\frac{\lambda}{1-\lambda}(1-t) b\right) d t \\
+f(b) \lambda(1-\lambda) \int_{0}^{1}(1-t) \sqrt{t} \sqrt{1-t} g\left(t a+\frac{\lambda}{1-\lambda}(1-t) b\right) d t
\end{gathered}
$$




$$
\begin{aligned}
& \leq \frac{1}{2}\left(\lambda^{2} \int_{0}^{1} t^{2} d t f(a) g(a)+\lambda(1-\lambda)(f(a) g(b)+f(b) g(a))\right) \\
&+\frac{1}{2}\left(\int_{0}^{1} t(1-t) d t+(1-\lambda)^{2} f(b) g(b) \int_{0}^{1}(1-t)^{2} d t\right) \\
&+2 \lambda^{2} \int_{0}^{1} t(1-t) f\left(t a+\frac{\lambda}{1-\lambda}(1-t) b\right) g\left(t a+\frac{\lambda}{1-\lambda}(1-t) b\right) d t
\end{aligned}
$$

By using $x=t a+\frac{\lambda}{1-\lambda}(1-t) b, d x=\left(a-\left(\frac{\lambda}{1-\lambda}\right) b\right) d t$ in (2.23), we obtain

$$
\begin{aligned}
& g(a) \lambda^{2} \int_{0}^{1} t \sqrt{t} \sqrt{1-t} f\left(t a+\frac{\lambda}{1-\lambda}(1-t) b\right) d t \\
&= g(a) \frac{\lambda^{2}}{\left(\left(\frac{\lambda}{1-\lambda}\right) b-a\right)^{3}} \int_{a}^{\left(\frac{\lambda}{1-\lambda}\right) b}\left(\left(\frac{\lambda}{1-\lambda}\right) b-x\right)^{\frac{3}{2}}(x-a)^{\frac{1}{2}} f(x) d x \\
& g(b) \lambda(1-\lambda) \int_{0}^{1}(1-t) \sqrt{t} \sqrt{1-t} f\left(t a+\frac{\lambda}{1-\lambda}(1-t) b\right) d t \\
&= g(b) \frac{\lambda(1-\lambda)}{\left(\left(\frac{\lambda}{1-\lambda}\right) b-a\right)^{3}} \int_{a}^{\left(\frac{\lambda}{1-\lambda}\right) b}\left(\left(\frac{\lambda}{1-\lambda}\right) b-x\right)^{\frac{1}{2}}(x-a)^{\frac{3}{2}} f(x) d x \\
& f(a) \lambda^{2} \int_{0}^{1} t \sqrt{t} \sqrt{1-t} g\left(t a+\frac{\lambda}{1-\lambda}(1-t) b\right) d t \\
&= f(a) \frac{\lambda^{2}}{\left(\left(\frac{\lambda}{1-\lambda}\right) b-a\right)^{3}} \int_{a}^{\left(\frac{\lambda}{1-\lambda}\right) b}\left(\left(\frac{\lambda}{1-\lambda}\right) b-x\right)^{\frac{3}{2}}(x-a)^{\frac{1}{2}} g(x) d x \\
& f(b) \lambda(1-\lambda) \int_{0}^{1}(1-t) \sqrt{t} \sqrt{1-t} g\left(t a+\frac{\lambda}{1-\lambda}(1-t) b\right) d t \\
&= f(b) \frac{\lambda(1-\lambda)}{\left(\left(\frac{\lambda}{1-\lambda}\right) b-a\right)^{3}} \int_{a}^{\left(\frac{\lambda}{1-\lambda}\right) b}\left(\left(\frac{\lambda}{1-\lambda}\right) b-x\right)^{\frac{1}{2}}(x-a)^{\frac{3}{2}} g(x) d x \\
& \int_{0}^{1} t^{2} d t=\int_{0}^{1}(1-t)^{2} d t=\frac{1}{3}, \int_{0}^{1} t(1-t) d t=\frac{1}{6} .
\end{aligned}
$$

Hence, this completes the proof.

\section{Acknowledgment}

The authors are supported by The Research Group in Mathematics and Applications at the University of Lagos, Nigeria.

\section{References}

[1] Ardic, M.A and Ozdemir, M.E., On some inequalities for different kinds of convexity. J. Inequal. Appl. 3 (2012), 1-6.

[2] Berwein, J. M and Vanderwerff, J. D., Convex Functions:Constructions, Characterizations and Counterexamples. Cambridge University Press (2010), ISBN 978-0-521-85005-6.

[3] Dragomir S. S., Pecaric J. and Persson L.E., Some inequalities of Hadamard type. Soochow J. Math. 21 (1995), $335-241$.

[4] Dragomir, S. S., and Toader, G., Some Inequalities for $m$-convex functions. Studia Univ. Babes-Bolyai Math. 38 (1993), no. 1, 21-28. 
[5] Fenchel, W., Convexity through the ages. Proceedings Convexity and its Applications (1983), 120-130.

[6] Godunova E. K. and Levin V. I., Neravenstva dlja funkcii sirokogo klassa, soderzascego vypuklye monotonnye i nekotorye drugie vidy funkii. Vycisli-tel. Mat. i. Fiz. Mezvuzov. Sb. Nauc. Trudov, MGPI, Moskva (1985), 138-142.

[7] Hadamard J., Etude Sur les properties des fontions entieres et en particular d'une function consideree par Riemann. J. Math. Pure and Appl. 58 (1893), 171-215.

[8] Omotoyinbo O. and Mogbademu A., On some Hadamard inequality for Godunova-Levin and MT-Convex functions. J. Nig. Assoc. Math. Phys. 25 (2013), no: II, 215-222.

[9] Omotoyinbo, O. and Mogbademu, A., Some New Hermite-Hadamard Integral inequalities for convex functions. Int. J. Sci. Innovation Tech. 1 (2014), no. 1, 001-012.

[10] Ozdemir, M.E., Ekinci, A and Akdemir, A.O., Some new Integral Inequalites for several kinds of convex functions. Arxiv.org/pdf/1202.2003. (2012), 1-11.

[11] Tunc M., and Yildirim, H., On MT-convexity. http:/ /arxiv.org/pdf/1205.5453.pdf. (2012), Preprint.

[12] Tunc M., Subas Y., and Karabayir I., On some Hadamard type inequalities for MT-convex functions. Int. J. Open Problems Comp. Math. 6 (2013), no. 2, 1998-6262.

\section{Affiliations}

OPEYEMI OMOTOYINBO

ADDRESS: University of Lagos, Dept. of Mathematics, Lagos-Nigeria.

E-MAIL: opetoyinbo@yahoo.com

Adesanmi Mogbademu

ADDRESS: University of Lagos, Dept. of Mathematics, Lagos-Nigeria.

E-MAIL: amogbademu@unilag.edu.ng

Peter Olanipekun

AdDress: University of Lagos, Dept. of Mathematics, Lagos-Nigeria.

E-MAIL: polanipekun@yahoo.com 